\author{
Alterstice \\ Revue internationale de la recherche interculturelle \\ International Journal of Intercultural Research \\ Revista International de la Investigacion Intercultural
}

\title{
Le retour au travail d'immigrants ayant subi une lésion professionnelle : les embûches de la rencontre interculturelle et la précarité du lien d'emploi
}

\author{
Sylvie Gravel, Jessica Dubé, Daniel Côté, Bob W. White et Danielle Gratton
}

Volume 7, numéro 1, 2017

L’interculturel dans la Cité : actes à poser en contexte pluriethnique

URI : https://id.erudit.org/iderudit/1040609ar

DOI : https://doi.org/10.7202/1040609ar

Aller au sommaire du numéro

Éditeur(s)

Alterstice

ISSN

1923-919X (numérique)

Découvrir la revue

Citer cet article

Gravel, S., Dubé, J., Côté, D., White, B. W. \& Gratton, D. (2017). Le retour au travail d'immigrants ayant subi une lésion professionnelle : les embûches de la rencontre interculturelle et la précarité du lien d'emploi. Alterstice, 7(1), 21-38. https://doi.org/10.7202/1040609ar
Résumé de l'article

Cet article rapporte une partie des résultats d'une étude exploratoire sur le processus de réadaptation et de retour au travail en contexte de relations interculturelles. Cette étude avait deux objectifs : identifier les stratégies mises de l'avant par les différents acteurs pour faciliter le processus de réadaptation et de retour au travail des travailleurs immigrants et décrire les contraintes, les obstacles et les aides au processus selon la perspective des différents acteurs. Des entrevues semi-dirigées ont ainsi été menées auprès de 47 répondants : cliniciens, agents d'indemnisation et conseillers en réadaptation de la CNESST, employeurs et travailleurs immigrants victimes d'une lésion reconnue et indemnisée. Au-delà des barrières linguistiques et culturelles, deux facteurs nuisent au retour en emploi des travailleurs immigrants : d'une part, la précarité du lien d'emploi freine leurs efforts et mine leur volonté de consolider leur état de santé et, d'autre part, la menace ou la perte réelle du lien d'emploi amène une rupture symbolique dans leur processus d'intégration. Cette rupture est importante pour ceux qui sont surqualifiés pour leur poste, surtout si leur emploi est un passage obligé pour acquérir une expérience de travail au Canada et viser ensuite un emploi à la hauteur de leurs compétences. Le processus de réadaptation peut soit se transformer en un " parcours déshonorant ", soit fournir une occasion d'améliorer sa situation sur un marché du travail discriminant. Adapter les programmes à la réalité de ces travailleurs immigrants est possible, mais pose un dilemme moral et éthique : comment motiver les travailleurs à se rétablir tout en explorant un autre créneau d'emploi si leur lien d'emploi risque d'être rompu, alors que les règles relatives au droit de retour au travail prévoient la réintégration de l'emploi prélésionnel ou tout autre poste jugé équivalent?
Droits d'auteur (C) Sylvie Gravel, Jessica Dubé, Daniel Côté, Bob W. White et Danielle Gratton, 2017
Ce document est protégé par la loi sur le droit d'auteur. L’utilisation des services d'Érudit (y compris la reproduction) est assujettie à sa politique d'utilisation que vous pouvez consulter en ligne.

https://apropos.erudit.org/fr/usagers/politique-dutilisation/ 


\section{7}

ARTICLE THÉMATIQUE

\section{Le retour au travail d'immigrants ayant subi une lésion professionnelle : les embûches de la rencontre interculturelle et la précarité du lien d'emploi}

Sylvie Gravel ${ }^{1}$, Jessica Dubé ${ }^{2}$, Daniel Côté ${ }^{3}$, Bob W. White ${ }^{2}$ et Danielle Gratton ${ }^{4}$

\section{Résumé}

Cet article rapporte une partie des résultats d'une étude exploratoire sur le processus de réadaptation et de retour au travail en contexte de relations interculturelles. Cette étude avait deux objectifs : identifier les stratégies mises de l'avant par les différents acteurs pour faciliter le processus de réadaptation et de retour au travail des travailleurs immigrants et décrire les contraintes, les obstacles et les aides au processus selon la perspective des différents acteurs. Des entrevues semi-dirigées ont ainsi été menées auprès de 47 répondants : cliniciens, agents d'indemnisation et conseillers en réadaptation de la CNESST, employeurs et travailleurs immigrants victimes d'une lésion reconnue et indemnisée. Au-delà des barrières linguistiques et culturelles, deux facteurs nuisent au retour en emploi des travailleurs immigrants : d'une part, la précarité du lien d'emploi freine leurs efforts et mine leur volonté de consolider leur état de santé et, d'autre part, la menace ou la perte réelle du lien d'emploi amène une rupture symbolique dans leur processus d'intégration. Cette rupture est importante pour ceux qui sont surqualifiés pour leur poste, surtout si leur emploi est un passage obligé pour acquérir une expérience de travail au Canada et viser ensuite un emploi à la hauteur de leurs compétences. Le processus de réadaptation peut soit se transformer en un "parcours déshonorant ", soit fournir une occasion d'améliorer sa situation sur un marché du travail discriminant. Adapter les programmes à la réalité de ces travailleurs immigrants est possible, mais pose un dilemme moral et éthique : comment motiver les travailleurs à se rétablir tout en explorant un autre créneau d'emploi si leur lien d'emploi risque d'être rompu, alors que les règles relatives au droit de retour au travail prévoient la réintégration de l'emploi prélésionnel ou tout autre poste jugé équivalent?

\section{Rattachement des auteurs}

${ }^{1}$ Institut Santé et société, Université du Québec à Montréal, Montréal, Canada; ${ }^{2}$ Université du Québec à Montréal, Montréal, Canada ; ${ }^{3}$ Institut de recherche Robert Sauvé en santé et sécurité au travail, Montréal, Canada; ${ }^{4}$ Centre intégré de santé et de services sociaux (CIUSS) Laval, Montréal, Canada

\section{Correspondance}

gravel.s@uqam.ca

\section{Mots clés}

travailleurs immigrants victimes de lésions professionnelles

\section{Pour citer cet article}

Gravel, S., Dubé, J., Côté, D., White, B. W. et Gratton, D. (2017). Le retour au travail d'immigrants ayant subi une lésion professionnelle : les embûches de la rencontre interculturelle et la précarité du lien d'emploi. Alterstice, 7(1), 21-38. 


\section{Introduction}

Les taux d'abandon des programmes de réadaptation de la douleur chronique rapportés dans la littérature sont plus élevés chez les personnes immigrantes (Sloots, Dekker, Bartels, Geertzen et Dekker, 2011). Ces abandons seraient liés à la qualité de la relation thérapeutique entre les travailleurs, les médecins et les physiothérapeutes (Sloots, Dekker, Pont, Bartels, Geertzen et Dekker, 2010). Selon Sloots et coll. (2010), les barrières linguistiques limiteraient la communication et les barrières culturelles influenceraient les attentes et les responsabilités partagées entre le patient, le thérapeute et l'employeur pour réadapter et réinsérer professionnellement le travailleur.

Au Québec, les données permettant de calculer les taux d'abandon des immigrants inscrits aux programmes de retour au travail ne sont pas colligées. La Commission des normes du travail, de l'équité et de la santé et de la sécurité au travail (CNESST) ${ }^{1}$ qui gère ces fichiers veut éviter une utilisation inappropriée sur le plan légal, voire discriminante, de telles données. Néanmoins, grâce à des observations empiriques faites par les conseillers en réadaptation à l'emploi de la CNESST, les administrateurs de ces programmes ainsi que les cliniciens (physiothérapeutes, ergothérapeutes et kinésiologues) en soutien au processus de réadaptation et de réintégration au travail, on sait qu'il existe de multiples et complexes barrières dans les dossiers des travailleurs immigrants.

Ces observations s'inscrivent dans une réflexion plus large faite au Québec (Gravel et coll., 2016), au Canada (Premji, Duguay, Messing et Lippel, 2010; Vosko, 2009) et dans bien des pays européens (Ori et Sargeant, 2011) et de l'Océanie (Quinlam, 2011) sur les lésions professionnelles chez les travailleurs en situation de vulnérabilité, parmi lesquels les travailleurs immigrants sont surreprésentés au sein de la population de travailleurs. Au Québec, les travailleurs immigrants en quête d'une première expérience canadienne de travail trouveront très souvent des emplois dans des agences de location de personnel (Commission des normes du travail au Québec, 2012; Vulture et Provencher, 2014) ou dans des petites manufactures non syndiquées (Gravel, Legendre, Rhéaume, Séguin et Gagné, 2013). On constate que les travailleurs immigrants, généralement plus scolarisés que leurs collègues originaires du Canada, sont plus exposés aux risques de lésions et moins bien formés et supervisés, parce que les employeurs considèrent l'encadrement comme superflu pour des travailleurs de passage dans leur entreprise (Dubé et Gravel, 2014; Gravel et coll., 2016). Malgré tous ces travaux empiriques, la surveillance des lésions professionnelles chez les travailleurs immigrants en fonction de l'origine des travailleurs ou de la précarité des liens d'emploi, et ce, pour les agences comme pour les entreprises agricoles, n'est pas encore établie (Gravel et coll., 2016; Massé, 2016).

Face à ces observations, les services de réadaptation de Montréal pour les travailleurs ayant subi une lésion professionnelle ont sollicité notre équipe de recherche pour explorer ce thème et tenter de répondre aux questions des conseillers en réadaptation. Quels sont les contraintes et les facteurs facilitant la réadaptation et la réinsertion au travail dans des contextes d'intervention interculturelle? Comment se réalise la démarche de réadaptation alors que le travailleur immigrant est encore en mode d'insertion sur le marché du travail? Est-il possible d'adapter en toute équité les programmes de réadaptation aux travailleurs immigrants?

\section{État des connaissances}

Lederer, Loisel, Rivard et Champagne (2014) ont recensé, entre 1981 et 2011, 423 publications traitant des modèles de réadaptation, illustrant l’ampleur des transformations de tels modèles au fil des années. Au début, le paradigme biomédical était au cœur de ces modèles, pour ensuite laisser place à des modèles plus complexes tenant compte des dimensions individuelles, organisationnelles et sociétales (Côté, 2014). Malgré tous ces raffinements, aucun de ces modèles ne s'est intéressé aux parcours d'insertion professionnelle des travailleurs immigrants et de leurs effets sur le processus de réadaptation. Au mieux, la relation interculturelle a été abordée sous l’angle des écarts culturels dans la relation interpersonnelle entre le clinicien et le travailleur, négligeant la dimension collective de la

\footnotetext{
${ }^{1}$ Lors de la réalisation de cette étude, cette organisation portait le nom de Commission de la santé et de la sécurité du travail (CSST). Elle est devenue la CNESST à la suite de sa fusion avec la Commission des normes du travail (CNT) et la Commission de l'équité salariale (CES).
} 
réintégration du travailleur sur le marché du travail, et seulement en envisageant l'environnement de travail prélésionnel.

Les modèles biomédicaux des années 1970 étaient centrés sur le dysfonctionnement du travailleur dans ses activités professionnelles et sur l'amplitude de ses limites (Schultz, Stowell, Feurstein et Gatchel, 2007). Les stratégies de réadaptation misaient sur la rencontre thérapeutique travailleur-médecin-physiothérapeute pour surmonter les limitations fonctionnelles du travailleur sans égard à la réalité interculturelle (Schultz et coll., 2007). Une décennie plus tard, les modèles misaient sur les stratégies pour soulager les douleurs chroniques des travailleurs, entre autres sur la perception de la douleur (Waddell, 1987, cité par Loisel et coll., 2005). Par la suite, la neuropsychologie et la psychologie ont contribué au développement d'approches psychosociales de la réadaptation centrées sur les épisodes de douleur envahissante et désorganisant l'identité du travailleur, contribuant ainsi à faire tomber le préjugé que "la douleur est dans la tête des travailleurs " (traduction libre de Schultz et coll., 2007). Enfin, les modèles se sont intéressés aux dimensions organisationnelles, dont les coûts directs et indirects de la réadaptation, les coûts d'assurance (convalescence, soins, réadaptation psychologique) et l'évaluation des pertes pécuniaires de ces limitations (perte d’un membre, de la mobilité, de la jouissance de la vie) (Lederer et coll., 2014; Schultz et coll., 2007).

Aujourd'hui, les modèles intègrent les bienfaits du soutien de l'environnement du travailleur, en se basant sur le modèle d'écologie sociale qui place le travailleur au cœur d'un système impliquant la famille, les proches (microsystème), les services d'indemnisation, de réadaptation (mésosystème), le système économique et de sécurité sociale et le marché du travail (macrosystème) (Lederer et coll., 2014; Schultz et coll., 2007). Ainsi, l'idée que toute modification du contexte social peut affecter le processus de réadaptation en dehors de la relation thérapeutique patient-soignant fait désormais partie intégrante du modèle de réadaptation au travail, souvent assimilé au paradigme de l'incapacité (Loisel et coll., 2005). Selon Kristman et coll. (2016), les récents modèles d'analyse portent une attention particulière aux efforts physiques et psychologiques exigés des travailleurs comme aux éléments stressants propres à l'organisation du travail et aux valeurs de performance, qui peuvent être difficiles à surmonter pour les travailleurs souffrant de limitations fonctionnelles à la suite de leur lésion professionnelle (Côté et Coutu, 2010; Ockander et Timpka, 2003).

En suivant cette perspective organisationnelle et sociétale, MacEachen, Kosny, Ferrier et Chambers (2010) ont étudié les contraintes administratives du système d'indemnisation ontarien qui perturbent le processus de réadaptation. Ils ont dégagé trois facteurs : a) l'absence d'obligation des employeurs à aménager un poste pour les travailleurs ayant subi une lésion professionnelle, situation qui mène trop souvent à la perte du lien d'emploi, b) la pression exercée par le système d'indemnisation auprès des médecins traitants afin qu'ils fournissent une expertise multidisciplinaire fine, coûteuse et complexe pour les travailleurs et c) les lacunes de communication induites par les services administratifs de l'indemnisation (missives rédigées en termes juridiques, entrevues sans interprète) qui sèment la confusion et privent le travailleur de la pleine maîtrise du processus. Ces travaux réalisés en Ontario concordent, en plusieurs points, avec les observations faites sur l'accès à l'indemnisation des travailleurs immigrants, démontrant que les obstacles à l'accès sont liées aux contraintes administratives, juridiques, médicales et aux conditions de retour au travail à la suite d'une lésion professionnelle (Gravel et coll., 2016).

D'autres auteurs ont documenté la crainte de perdre le lien d'emploi comme étant le principal facteur nuisant au processus de réadaptation (Lysaght et Larmour-Trode, 2008; Sjöström, Melin-Johansson, Asplund et Alricsson, 2011; Young, 2010). Selon Young (2010), si le travailleur est assuré de conserver son lien d'emploi, au-delà du seul souci de préserver ses revenus, sa réadaptation progressera rapidement. Lysaght et Larmour-Trode (2008) ont observé que les éléments les plus importants pour compléter avec succès un programme de réadaptation sont, pour le travailleur, le soutien émotionnel des collègues, le soutien technique des ressources humaines pour préparer correctement son dossier de réclamation et le fait de recevoir de son superviseur des commentaires positifs sur sa progression. Ces conditions supposent qu'un responsable des ressources humaines ou un superviseur joue un rôle central pour soutenir le travailleur dans son processus de réadaptation et de retour au travail (Higgins, O'Halloranet Porter, 2012; Lysaght et Larmour-Trode, 2008). II s'agit de conditions optimales auxquelles peu de travailleurs immigrants ont accès au cours de leurs premières années d'établissement (Gravel et Dubé, 2016). 
Pour Dressler et Pils (2009) et Premji (2015), les barrières linguistiques et culturelles nuisent à la communication et deviennent un fardeau pour tous les acteurs impliqués dans le processus de réadaptation. Le travailleur est frustré de ne pas pouvoir communiquer aisément sa souffrance, et les thérapeutes éprouvent des difficultés à faire comprendre les objectifs thérapeutiques et à offrir un traitement adapté (Niemeier, Burnett et Whitaker, 2003). Pour les proches jouant le rôle d'interprète, la situation devient pénible lorsque des tensions s'installent entre le travailleur, les thérapeutes et le conseiller en réadaptation (Dressler et Pils, 2009). Pour surmonter ces barrières, Sloots et coll. (2011) suggèrent aux établissements d'implanter des programmes de contrôle de la douleur adaptés en augmentant la durée et la fréquence des rencontres cliniques tout en misant sur la formation interculturelle des cliniciens.

Pour Premji (2015), le problème de communication prend une ampleur considérable lors du retour au travail pour les travailleurs immigrants ne maîtrisant pas bien les langues officielles. Les possibilités de retour au travail sont réduites, voire quasi inexistantes, si les employeurs, souvent issus de la même communauté culturelle que les travailleurs immigrants, ne sont pas en mesure de reprendre le travailleur à cause de ses limitations fonctionnelles (Premji, 2015). Les autres employeurs potentiels pouvant offrir un emploi convenable pourraient en toute légitimité imposer la maîtrise des langues officielles (Premji, 2015). Toutefois, la mise à niveau des langues pour améliorer l'accès au marché du travail est une mesure exceptionnelle qui s'éloigne de l'esprit actuel de la Loi sur les accidents du travail et les maladies professionnelles (LAMTP, chapitre A-3.001), qui vise à réintégrer le travailleur sur le marché du travail dans un emploi jugé équivalent à l'emploi prélésionnel' ${ }^{2}$. Une telle adaptation des programmes à la réalité des travailleurs immigrants pose un dilemme de gestion important au moment de développer des approches optimales de réadaptation tenant compte des lacunes, mais aussi des compétences des travailleurs, qui peuvent être différentes, voire supérieures à celles exigées par le poste occupé lors de la survenue de la lésion (Lederer et coll., 2014).

Malgré l'exhaustivité des écrits sur les modèles d'analyse, aucun de ces modèles n'aborde le rôle de la précarité du lien d'emploi sur la motivation des travailleurs immigrants à se rétablir sur le plan physique et psychologique, une précarité qui, dans certains cas, se transforme en une rupture symbolique avec le processus de réinsertion sur le marché du travail. En s'intéressant à la précarité du lien d'emploi comme facteur nuisant au processus de réadaptation, il est possible de bonifier et de raffiner les modèles d'analyse au-delà des dimensions culturelle et symbolique du travail et de la douleur (Côté, Gratton, Gravel et Dubé, 2015).

Le concept de rupture symbolique a été développé par le neurologue Edelman (2003, cité par Denizeau, 2013) et repris par divers précurseurs de la philosophie et de l'anthropologie, qui proposent de concevoir nos expériences du monde comme un élément qui façonne notre corps et notre perception du monde, dans un sens non mythologique mais psychosomatique. Denizeau (2013) soutient qu' « avoir mal, c'est encore ressentir l'agression du monde et la menace qu'il constitue ». La rupture symbolique est un concept fréquemment mobilisé par les médiateurs interculturels mis à contribution dans les suivis de cas pour dénouer les impasses thérapeutiques d'interventions faites en milieu hospitalier (Bouznah et Lewertowski, 2014).

\section{Cadre d'analyse}

L'ensemble des concepts mobilisés pour mener cette étude a été organisé dans un modèle qui conjugue à la fois le modèle d'analyse du cumul de précarités chez les travailleurs immigrants ayant subi une lésion professionnelle, ceux de la souffrance physique et psychologique des travailleurs et celui de la rupture symbolique. Cet ensemble de concepts est illustré dans la figure 1.

\footnotetext{
${ }^{2}$ Article 236 : Le travailleur ayant subi "une lésion professionnelle qui redevient capable d'exercer son emploi a droit de réintégrer prioritairement son emploi dans l'établissement où il travaillait lorsque s'est manifestée sa lésion ou de réintégrer un emploi équivalent dans cet établissement ou dans un autre établissement de son employeur". (c'est nous qui soulignons) 1985, c. 6, a. 236.
} 
Figure 1

Effets du cumul de précarités du lien d'emploi sur le rétablissement des travailleurs immigrants ayant subi une lésion professionnelle

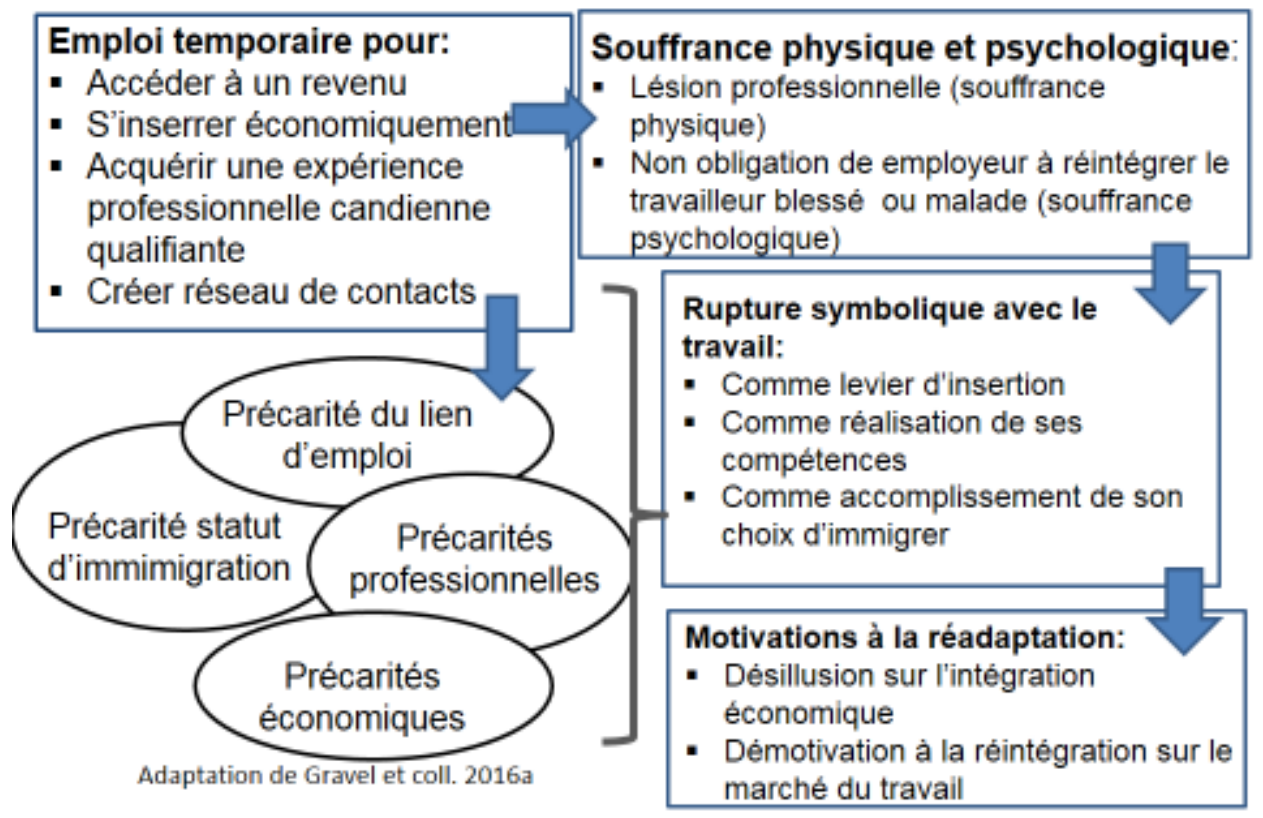

Le cumul de précarité comprend quatre dimensions, dont chacune intègre diverses situations spécifiques :

a) Précarité du lien d'emploi, qui distingue les contrats permanents ou temporaires (comme les contrats avec une agence ou un sous-traitant) des situations de précarité aggravées (lorsqu'il y a une cascade d’intermédiaires entre l'entreprise cliente et le travailleur exécutant les tâches).

b) Précarité du statut d'immigration, pour les travailleurs n'ayant pas encore obtenu leur résidence permanente, les travailleurs étrangers temporaires, les réfugiés et ceux en attente du statut de réfugié.

c) Précarité professionnelle, qui inclut la non-reconnaissance des diplômes et des expériences professionnelles acquis à l'étranger ainsi que la non-reconnaissance de l'ancienneté accumulée dans l'entreprise ou dans le métier.

d) Précarité économique, qui fait référence au cumul des heures travaillées sans reconnaissance et des heures supplémentaires non rémunérées, au taux horaire au salaire minimum sans égard aux expériences et compétences, à l'endettement des travailleurs pour être inscrits sur la liste des candidats au recrutement et aux obligations financières envers la famille restée outremer, dont certains membres dépendent du revenu du travailleur pour subvenir à leurs besoins.

Dans le cadre de notre étude, nous avons porté une attention particulière aux liens entre la rupture symbolique avec le travail et les diverses dimensions du cumul de précarité comme éléments déterminant de cette rupture.

\section{Méthodologie}

Initialement, 65 répondants ont été sollicités pour réaliser cette étude, représentant quatre groupes: a) les cliniciens, b) les agents d'indemnisation et conseillers en réadaptation, c) les employeurs et d) les travailleurs. Le taux de participation global a été de $72 \%$, mais il est non proportionnel : nous avons obtenu une participation parfaite des cliniciens (ergothérapeutes, physiothérapeutes et kinésiologues) (15/15) et la participation des agents d'indemnisation et des conseillers en réadaptation a été supérieure à celle souhaitée (21/15), mais celle des employeurs (2/15) et des travailleurs ayant subi une lésion professionnelle (9/15) n'a pas atteint l'objectif ciblé. II 
s'agit bien d'un échantillon raisonné qui ne prétend pas être représentatif. Néanmoins, nous considérons que nous avons atteint un seuil acceptable de saturation avec les entrevues menées auprès des cliniciens $(n=15)$ et des conseillers en réadaptation $(n=21)$, un seuil un peu moins fiable avec les travailleurs $(n=9)$ et aucunement fiable avec les employeurs $(n=2)$.

Les cliniciens ont été recrutés soit par l'intermédiaire de quatre cliniques privées pour lesquelles ils travaillaient depuis au moins une année, soit par leurs ordres ou associations professionnelles. Les professionnels de la réadaptation de la CNESST ont été recrutés par l'intermédiaire de la Direction de l'indemnisation et de la réadaptation des principaux districts administratifs de la grande région montréalaise de la CNESST. Ils devaient être employé par ces services depuis au moins une année et desservir une clientèle multiethnique. Ces deux processus de recrutement ont intentionnellement introduit un biais de sélection, celui d'avoir recruté des cliniciens ayant une longue expérience d'intervention auprès des travailleurs immigrants. Les professionnels de la réadaptation recrutés, que ce soit les cliniciens ou les conseillers en réadaptation, étaient majoritairement des hommes, âgés de 20 à 40 ans, canadiens ou européens de naissance et dont plus de la moitié de la clientèle était issue de l'immigration (tableau 1). Les prénoms des cliniciens et des conseillers en réadaptation associés aux transcriptions des entrevues dans la section des résultats sont fictifs, empêchant toute association avec une entreprise existante.

Tableau 1. Caractéristiques des répondants professionnels de la réadaptation

\begin{tabular}{|l|l|l|l|}
\hline & $\begin{array}{l}\text { Clinicien / cliniques } \\
\text { privées }\end{array}$ & $\begin{array}{l}\text { Conseiller } \\
\text { réadaptation/ } \\
\text { CNESST }\end{array}$ & $\begin{array}{l}\text { Pourcentage } \\
\text { total (\%) } \\
(\mathrm{n}=29)\end{array}$ \\
\hline Sexe & 9 & 11 & 69 \\
Homme & 6 & 3 & 31 \\
Femme & & & 35 \\
\hline Âge & 6 & 4 & 35 \\
20 à 29 ans & 4 & 6 & 10 \\
20 à 39 ans & 2 & 1 & 20 \\
40 à 49 ans & 3 & 3 & \\
50 ans et plus & & & 76 \\
\hline Pays d'origine & 10 & 12 & 14 \\
Canada & 4 & 0 & 2,5 \\
Europe & 1 & 0 & 4,5 \\
Asie de l'Est & 0 & 2 & 61,5 \\
Amérique du Sud & 62 & 61 & \\
\hline Pourcentage moyen & & & \\
de clientèle immigrante (\%) & &
\end{tabular}

La sollicitation des travailleurs a emprunté aussi la filiale des cliniques privées de réadaptation œuvrant dans la région métropolitaine de Montréal, ainsi que celle d'une association assurant la défense des travailleurs accidentés. Les critères d'inclusion des travailleurs étaient : a) au moment de l'enquête, avoir connu une absence prolongée du travail de plus de 90 jours depuis l'ouverture et l'acceptation du dossier d'indemnisation à la CNESST, b) avoir suivi un programme de réadaptation (ergothérapie, physiothérapie et kinésiologie), c) parler anglais ou français ou avoir accès à un interprète lors de l'entretien et $d$ ) se considérer comme une personne immigrante. Tous les noms et prénoms inscrits dans le tableau 2 qui suivent, de même que les noms des entreprises participantes et ceux associés aux entrevues, sont fictifs. 
Tableau 2. Caractéristiques des répondants travailleurs

\begin{tabular}{|c|c|c|c|c|c|}
\hline $\begin{array}{l}\text { Pseudony } \\
\text { me }\end{array}$ & Profession & Domaine d'études & Sexe & Âge & Région d'origine \\
\hline Amine & Journalier & $\begin{array}{l}\text { Droit, criminologie (M. Sc.), formation } \\
\text { dans le pays d'origine }\end{array}$ & $\mathrm{H}$ & 36 & Afrique du Nord \\
\hline Carmen & $\begin{array}{l}\text { Préposée aux } \\
\text { bénéficiaires }\end{array}$ & $\begin{array}{l}\text { Orthophonie (baccalauréat) } \\
\text { formation dans le pays d'origine }\end{array}$ & $\mathrm{F}$ & $\mathrm{n} / \mathrm{d}$ & Amérique du Sud \\
\hline Umberto & $\begin{array}{l}\text { Mécanicien } \\
\text { automobile }\end{array}$ & Mécanique, formation au Québec & $\mathrm{H}$ & 50 & Europe de l'Ouest \\
\hline Belkacem & $\begin{array}{l}\text { Préposée aux } \\
\text { bénéficiaires }\end{array}$ & $\begin{array}{l}\text { Soins infirmiers (baccacalauréat, } \\
\text { spécialisation en bloc opératoire) }\end{array}$ & $\mathrm{H}$ & - & Afrique du Nord \\
\hline Valencia & $\begin{array}{l}\text { Infirmière } \\
\text { auxiliaire }\end{array}$ & Infirmière auxiliaire, formation au Québec & $\mathrm{F}$ & 49 & Antilles \\
\hline Harica & Cuisinière & $\begin{array}{l}\text { Tech. en administration, formation dans le } \\
\text { pays d'origine }\end{array}$ & $\mathrm{F}$ & 50 & Proche-Orient \\
\hline Leticia & Cuisinière & $\begin{array}{l}\text { Cuisine d'établissement, formation au } \\
\text { Québec }\end{array}$ & $\mathrm{F}$ & 55 & Amérique du Sud \\
\hline Rocio & Concierge & $\begin{array}{l}\text { Coupe et confection du cuir, formation au } \\
\text { Québec }\end{array}$ & $\mathrm{H}$ & 61 & Amérique Centrale \\
\hline Dario & Rembourreur & $\begin{array}{l}\text { Tech. en comptabilité, formation dans le } \\
\text { pays d'origine, et dessin industriel, } \\
\text { formation au Québec }\end{array}$ & $\mathrm{H}$ & 47 & Amérique centrale \\
\hline
\end{tabular}

Abréviations : $\mathrm{F}$, femme; $\mathrm{H}$, homme.

Les deux entreprises participantes ont été recrutées par l'intermédiaire de l'équipe de santé au travail du CIUSSS du Centre-Ouest-de-l'île-de-Montréal (anciennement CSSS de la Montagne).

Les entretiens semi-dirigés (environ 90 minutes) ont été menés à l'aide d'un guide d'entrevue traitant des thèmes suivants : 1) moments-clés du processus de réadaptation, 2) parcours médical et de réadaptation, 3) rencontre interculturelle, 4) ressources disponibles pour accompagner les travailleurs immigrants, 5) formation interculturelle et 7) retour au travail et maintien du lien d'emploi. Les guides ont été adaptés au type de répondant, tout en préservant l'ensemble des thèmes. L'arborescence de codes a été construite à partir des sept thèmes de la grille d'entrevue. Les informations concernant les répondants ont été anonymisées, seul le statut professionnel ayant été conservé, pour repérer l'origine des propos.

Cet article traite du retour au travail et du maintien du lien d'emploi, les autres thèmes faisant l'objet de publications distinctes. Les entrevues ont été enregistrées, retranscrites et codées à l'aide du logiciel d'analyse qualitative NVivo 10.

Ce projet a fait l'objet d'une certification éthique délivrée par le Comité d'éthique de la recherche du CSSS de la Montagne ${ }^{3}$ et celui du Centre de recherche interdisciplinaire en réadaptation (CRIR) ${ }^{4}$ du Grand Montréal.

\footnotetext{
${ }^{3} \mathrm{~N}^{\circ} \mathrm{d}^{\prime}$ approbation R 879-10.04.2014. Chaque participant a signé un formulaire de consentement l'informant de la nature du projet et de sa participation, du déroulement du projet, des avantages et bénéfices, des risques et inconvénients, de la confidentialité, de la liberté de participation et de retrait et des engagements du chercheur.

${ }^{4} \mathrm{~N}^{\circ}$ d'approbation CRIR-970-0514.
} 


\section{Résultats}

Les résultats sont regroupés en trois thèmes. Le premier thème concerne les trajectoires de retour au travail chez les travailleurs immigrants : a) le retour possible (même employeur), b) le retour difficilement envisageable à cause de mauvaises relations avec l'employeur et c) le retour possible chez un autre employeur parce que le lien d'emploi initial est rompu. Le deuxième thème est constitué des difficultés de réadaptation et de leurs impacts sur le retour au travail. Quant au troisième thème, il regroupe les stratégies déployées par les cliniciens et les conseillers en réadaptation afin de surmonter les obstacles à la réadaptation et à la réintégration des travailleurs immigrants.

\section{Trajectoires de retour au travail}

Comparativement à l'ensemble des répondants, les conseillers en réadaptation ont répondu de façon plus explicite sur les trajectoires de retour au travail, parce qu'ils jouent un rôle primordial dans ce processus en mettant en place les ressources d'accompagnement adaptées à l'état de santé physique et psychologique du travailleur et au potentiel de réinsertion de ce dernier dans son milieu de travail ou dans un emploi équivalent. Lors de l'évaluation du potentiel de retour au travail, ils tiennent compte des interactions entre le travailleur ayant subi une lésion et le soutien de son milieu de travail, ce qui inclut la présence ou non d'un appui syndical, du soutien des collègues et de celui des supérieurs. Ils se réfèrent à un système de catégorisation des risques de chronicité chez les travailleurs pour créer leur plan d'intervention, système en vigueur depuis de nombreuses années au sein des services de réadaptation de la CNESST. Les trajectoires classées de 1 à 3 sont relativement simples à traiter, puisque le travailleur conserve son lien d'emploi. Ces travailleurs semblent bénéficier généralement de meilleures conditions de réintégration au travail parce qu'ils sont employés par de grandes entreprises syndiquées et que le syndicat a prévu des aménagements de poste pour les affectations temporaires. Toutefois, comme le souligne l'un des conseillers en réadaptation,

[...] même si le travailleur immigrant est syndiqué, souvent il ne sait pas qu'il peut recourir à son syndicat pour négocier son retour au travail, son aménagement de poste. (Charlie, 34, conseiller en réadaptation)

Pour le travailleur associé à la catégorie 4, le lien d'emploi est également conservé, mais demeure fragile à cause des limitations fonctionnelles qui l'empêchent d'occuper le même poste. Selon un répondant, les possibilités seraient encore plus minces lorsqu'il s'agit d'une petite entreprise non syndiquée, les postes disponibles étant limités. Les employeurs peuvent se montrer réticents à réintégrer progressivement un travailleur blessé ou malade, préférant plus souvent le réintégrer seulement s'il est complètement rétabli :

On appelle l'employeur pour notre prise en charge puis l'employeur dit non, il va le réintégrer que lorsqu'il sera capable d'assumer ses tâches régulières. (Juliette, 45 , conseillère en réadaptation)

Si le processus de réadaptation dépasse le délai prescrit par la loi permettant au travailleur ayant subi une lésion professionnelle d'exercer son droit de retour au travail - une ou deux années selon la taille de l'entreprise ${ }^{5}-$ l'employeur peut être libéré de son obligation légale de réintégrer le travailleur. Préoccupé par la productivité, l'employeur sera plus enclin à remplacer le travailleur en convalescence plutôt que de le maintenir en emploi, surtout s'il s'agit d'un poste non spécialisé.

Pour les travailleurs associés aux catégories 5, 6 et 7, le lien d'emploi est presque systématiquement perdu et, dans la majorité des cas, des atteintes physiques et psychiques sont visibles. La perte du lien d'emploi peut être attribuée à diverses raisons : la fermeture imminente de l'entreprise, ou des relations de travail distantes, comme le travail au sein d'une agence de placement de personnel ou d'une entreprise sous-traitante. Dans les deux

\footnotetext{
${ }^{5}$ LAMTP, article 240. Les droits conférés par les articles 236 à 239 peuvent être exercés : $1^{\circ}$ dans l'année suivant le début de la période d'absence continue du travailleur en raison de sa lésion professionnelle, s'il occupait un emploi dans un établissement comptant 20 travailleurs ou moins au début de cette période ou $2^{\circ}$ dans les deux ans suivant le début de la période d'absence continue du travailleur en raison de sa lésion professionnelle, s'il occupait un emploi dans un établissement comptant plus de 20 travailleurs au début de cette période.

1985 , c. 6, a. 240.
}

Alterstice - Revue Internationale de la Recherche Interculturelle, vol. 7, $n^{\circ} 1$ 
derniers cas, le travailleur sera trop souvent relégué au bas de la liste des travailleurs rappelés pour une affectation.

Parmi les cas les plus difficiles, les P4 et plus, $50 \%$ des travailleurs sont issus de l'immigration, et ce, encore qu'il y ait beaucoup de sous-déclarations chez les travailleurs immigrants. (Charlotte, 30, conseillère en réadaptation)

Ces cas difficiles nous [les conseillers en réadaptation] prennent $40 \%$ plus de temps à cause des problèmes de communication. (Clara, 59, conseillère en réadaptation)

\section{Difficultés de réadaptation et leurs retombées}

Si plusieurs difficultés surgissent lors du processus de réadaptation, la principale demeure, selon les conseillers en réadaptation et les travailleurs, la perte de revenu, et ce, même si le travailleur bénéficie d'une indemnisation :

Les travailleurs issus de l'immigration ont une mauvaise perception de leur arrêt de travail, qui se résume trop souvent à 90 \% d'un petit salaire. (Rosalie, 29, conseillère en réadaptation)

Il y a aussi la peur de perdre son travail ou d'être incapable de trouver un autre emploi convenable sur le marché du travail. Ces peurs, qui semblent universelles, selon les conseillers en réadaptation (Rosalie 29 et Charlie 34), chez tous les travailleurs occupant des emplois manuels non syndiqués ou offrant des conditions de travail précaires, ont des répercussions sur l'image de soi, sur les relations avec les collègues et avec les membres de la famille. Pour les travailleurs immigrants, les difficultés d'intégration à la société s'ajoutent à ces peurs. Elles s'inscrivent dans un continuum, dont les deux extrémités sont, comme les dénomme l'un des conseillers en réadaptation (Clara, 59, conseillère en réadaptation), le " parcours déshonorant » de réintégration sur le marché du travail et le parcours des "opportunités pour changer la situation d'emploi ». Dans ce continuum s'inscrivent des situations injustes pour lesquelles les conseillers tentent d'ajuster leur offre de services, afin d'accompagner les travailleurs vers le retour un travail sain et durable.

\section{Parcours déshonorant}

Selon les conseillers en réadaptation, chez le travailleur immigrant qui occupait, avant sa lésion, un emploi transitoire afin d'acquérir une expérience de travail en sol canadien - expérience nécessaire pour briguer un emploi à la hauteur de ses compétences - le processus de réintégration sur le marché du travail se transforme trop souvent en "parcours déshonorant». Ce déshonneur atteint le travailleur en tant que pourvoyeur de sa famille immédiate et de celle restée au pays. Pour certains conseillers en réadaptation et cliniciens, ce sentiment de déshonneur serait si fort que :

[...] ils vont des fois aller un petit peu plus rapidement que ce qu'ils devraient faire, ils vont essayer d'avoir moins de traitement, de retourner plus vite au travail [...] au risque de se blesser à nouveau, parce que c'est une honte d'être en arrêt de travail. (Charlie, 34, conseiller en réadaptation)

Ce déshonneur serait encore plus marquant si le travailleur demeure handicapé ou, pire, si cette lésion a des conséquences psychologiques.

[...] je n'avais jamais vu ça, des travailleurs qui se retrouvaient amputés recevaient des membres de la famille restés au pays un appui vraiment bizarre : ils les décourageaient de faire quoi que ce soit ou de surtout ne pas aller consulter en psychologie. [...] Les hommes en faisaient une blague entre eux. » (Clara, 59, conseillère en réadaptation)

Parmi les travailleurs immigrants suivis par les conseillers en réadaptation, et particulièrement les hommes, la psychothérapie prescrite pour favoriser le rétablissement du travailleur ayant subi une lésion professionnelle est mal perçue. 
[...] Dans beaucoup de cultures, le psychologue est considéré comme quelqu'un qui soigne des fous et qui est lui-même un peu fou. (Clara, 59, conseillère en réadaptation)

La psychothérapie est souvent vécue par les travailleurs comme une intrusion dans leur vie personnelle, les obligeant à se confier sur des événements antérieurs à leur lésion professionnelle, qu'ils ne veulent pas se remémorer, surtout s'ils sont liés à la guerre ou à de la violence organisée (torture, disparition, viol). Au contraire, pour les conseillers en réadaptation, connaître le parcours migratoire du travailleur est utile pour orienter leur action :

[...] ça serait aidant de connaître les statuts d'immigration à l'arrivée, pour faire en sorte que le processus de réadaptation des travailleurs puisse se dérouler correctement. (Clara, 59, conseillère en réadaptation)

Dans certaines sociétés, les professions dédiées à la relation d'aide comme les psychologues et les travailleurs sociaux sont méconnues. Selon les conseillers en réadaptation, certains travailleurs se plieront de bonne grâce à la psychothérapie dans l'espoir de soulager leurs douleurs chroniques, qui perturbent leur état d'esprit. Mais pour d'autres, la psychothérapie les éloigne de leurs attentes de retour rapide sur le marché du travail et de la représentation qu'ils ont de leur état de santé :

[Le thérapeute m'a suggéré de me projeter dans un endroit où je suis heureux, où je me sens bien]. J'aime la plage, toute ma vie c'est dans la plage et tout ça. Donc le seul endroit où je me sens bien à l'aise, c'est à la mer ou tout ça. [...] il a essayé un exercice avec moi et ça a réussi. [mais la réalité hivernale québécoise rattrape le patient] Maintenant, j'ai de la misère même à m'imaginer. " (Amine, 36, journalier, diplômé en droit dans son pays d'origine)

Si ce déshonneur est clairement nommé par les conseillers en réadaptation, les travailleurs sont peu enclins à s'en confesser. Toutefois, pour surmonter ce déshonneur, certains travailleurs se donnent comme mission d'accompagner d'autres travailleurs, pour éviter que la lésion qu'ils ont subie ne porte atteinte à leur dignité, pour s'assurer de ne pas être exploité par l'employeur, comme le rapporte ce même travailleur, employé par une agence de placement :

[...] j'essaie d'aider tous les amis qui sont à la CSST selon ma petite expérience au niveau des lois et tout [...] pour me conserver mes droits et tout ça. J'ai dit, je lui ai dit: est-ce que l'agence a déclaré l'accident? [Souvent] l'agence n'a pas déclaré son accident. (Amine, 36, journalier)

De façon générale, la sous-déclaration ou les incitatifs à celle-ci de la part des employeurs sont des injustices qui choquent maints travailleurs. À leurs yeux, c'est d'autant plus insidieux qu'ils ont été recrutés ou embauchés par des gens de leur communauté qui se disent leurs « frères » ou leurs « sœurs ":

L'agence qui m'a recruté embauche beaucoup d'Arabes parce que nous sommes intelligents, vites, [...] bons pour organiser les choses. [...] Mes études sont en droit, en criminologie [rien à voir avec le travail d'usine]. (Amine, 36, journalier)

Comme le souligne l'un des travailleurs, l'exploitation par des membres de sa propre communauté s'ajoute au déshonneur du parcours de réadaptation.

\section{Injustices ayant des impacts sur le processus de réadaptation}

Tout comme pour le déshonneur, ce sont les conseillers en réadaptation qui ont le mieux rapporté les situations perçues comme des injustices nuisant au processus de réadaptation. Parmi ces injustices, il a la lésion due à la négligence de l'employeur, pour laquelle l'employeur refuse de reconnaître sa responsabilité, et les limitations fonctionnelles permanentes qui nuisent à la quête du travailleur de trouver un meilleur emploi correspondant à ses compétences. Les deux premières injustices se rapportent aux comportements de l'employeur et la troisième à la détérioration irréversible de l'état de santé du travailleur. 
Nombre de travailleurs immigrants sont embauchés par une entreprise administrée ou appartenant à un membre du même groupe ethnoculturel. Le travailleur ayant subi une lésion professionnelle sera déçu de l'attitude de ses patrons, solidaires au moment de leur insertion sociale sur le marché du travail mais indifférents lorsque vient le temps de reconnaître le lien étiologique entre la lésion et les tâches exécutées :

[...] l'employeur conteste systématiquement tout, mais les travailleurs pensent que c'est contre eux, ils n'arrivent pas à comprendre qu'il y a des enjeux financiers dans la contestation [l'établissement du taux de cotisation à la CSST selon l'expérience de lésions dans l'entreprise, selon la fréquence et la gravité des lésions au cours des quatre dernières années]. (Rosalie, 29, conseillère en réadaptation)

Le sentiment d'injustice est très fort chez les travailleurs immigrants surqualifiés pour l'emploi occupé au moment de la lésion :

[...] des études qui n'étaient pas nécessairement reconnues ici, qu'ils ne savaient pas trop où aller. Donc ils sont arrivés ici, ils se sont trouvé un emploi, ils n'ont jamais fait les démarches pour que leur formation soit reconnue pour trouver un autre travail. Cette situation on l'a vue souvent. [...] Le sentiment d'injustice est encore plus grand quand ils n'aiment pas leur travail. (Rosalie, 29, conseillère en réadaptation)

Les réponses des travailleurs aux questions portant sur le sentiment d'injustice ont souvent été mêlées de propos rapportant des faits et des personnes, réponses qui ne permettaient pas de bien nommer les contextes et les objets d'injustice.

\section{Opportunité pour changer la situation d'emploi}

Selon les conseillers en réadaptation, certains travailleurs immigrants vont tenter de transformer cet arrêt de travail en une occasion pour changer leur situation d'emploi, et souvent dans le cas des femmes pour se consacrer à la famille. Les travailleurs surqualifiés vont réclamer que leurs diplômes et expériences acquises avant leur arrivée au Canada soient reconnus, afin de bénéficier d'un programme qui les oriente vers un créneau d'emploi proche de leurs compétences. Rappelons que dans le cas des professions exigeant un permis d'exercice ou des compétences spécifiques, les travailleurs immigrants n'exercant pas pendant quatre ou cinq ans leur profession ou leur métier peineront à faire reconnaître leurs compétences. La situation de déqualification professionnelle est un élément pouvant faire obstacle à une réelle démarche de réintégration, situation frustrante pour les travailleurs que rapportent bien des conseillers en réadaptation :

La non-reconnaissance des diplômes, c'est une difficulté. Reconnaissez-les et on ne partira pas à zéro tout le temps. Reconnaissez-les les diplômes qu'ils ont [...] sinon on va les payer pour le reste de leurs jours parce qu'on ne peut rien faire avec eux autres. C'est ridicule. (Zoé, 27 , conseillère en réadaptation)

Certains conseillers font des efforts pour concilier les compétences antérieures du travailleur ayant subi une lésion professionnelle et les besoins du marché du travail :

[...] maintenant, il y a trois options au niveau des études avec la CSST, qu'ils ont évalué que je veux faire : l'administration, la technique juridique et le travail social. C'est ça que je voulais faire, étudier. (Amine, 36, journalier)

La mise à niveau des compétences par une formation ajustée aux besoins du marché du travail du Québec n'est pas nécessairement une avenue adoptée par les services de réadaptation :

[...] l'agent de la CSST m'a trouvé un métier comme commis-comptabilité, puis j'ai pris une formation : 63 heures de théorie et 60 heures de stage. [...] j'étais tellement contente. Enfin, je peux travailler jusqu'à 75 ans, puis j'aurai un autre métier avec les chiffres. Je n'ai pas besoin de parler, les chiffres, 1, 2, 3, ce sont les mêmes dans le monde. [...] Mais tout à coup, après deux semaines, j'ai reçu une lettre, puis il m'envoyait [à] une autre agence pour recherche d'emploi comme commis de bureau. Tout de suite, j'ai appelé, puis j'ai contesté. » (Harica, 50, cuisinière) 
Selon l'un des conseillers en réadaptation et deux cliniciens, l'âge et l'usure prématurée due aux emplois ayant des contraintes physiques et des mouvements répétitifs vont ruiner la motivation des travailleurs immigrants. Cela est vécu comme un constat d'échec de leur intégration professionnelle :

[...] on a beaucoup de lésions chez des personnes de 55 ans et plus et encore plus chez les immigrants et particulièrement chez les femmes immigrantes. À partir de 55 ans, elles veulent prendre leur retraite, mais elles n'y ont pas droit. [...] à cet âge-là, les hommes comme les femmes se considèrent comme finis, puis il n'y a plus rien à faire avec eux autres [...]. Ça aussi, c'est une difficulté, très grande difficulté. (Zoé, 27, conseillère en réadaptation)

Retourner au travail pour être soumis aux mêmes contraintes et aux mêmes conditions de travail qu'avant la lésion rebute tous les travailleurs. C'est notamment le cas d'une travailleuse d'agence de placement de personnel spécialisée dans les soins de santé auprès de patients de centres hospitaliers de soins de longue durée (CHSLD) :

[...] malgré la douleur, il faut travailler pareil. Le patient est agité, il bouge. [...] j'ai peur de me faire mal davantage. [...]. Si j'avais le choix, mon Dieu, je changerais de travail, mais en attendant je ne peux pas rester chez moi. (Valencia, 49, infirmière auxiliaire)

S'ils perdent leur lien d'emploi et doivent chercher un nouvel emploi, un apprentissage est nécessaire, impliquant des efforts sous-estimés. C'est un processus auquel les travailleurs immigrants n'ont pas été souvent exposés, et encore moins s'il s'agit de leur premier emploi d'insertion :

[...] étant né ici, on sait comment ça marche se retrouver un emploi, tu dois faire un CV, rédiger une lettre de présentation, te présenter chez les employeurs, tu dois chercher sur Internet les offres d'emplois, tu dois faire des suivis téléphoniques. [...] Pour nous, $c^{\prime}$ est une évidence, mais pour eux, c'est un processus difficile à saisir. [...] C'est comme s'ils n'avaient jamais eu l'opportunité de s'ouvrir au reste en général. [...] Par exemple, dans les entreprises du textile, c'est un monde où les travailleuses et les travailleurs ont toujours fait un travail manuel, qu'ils n'aient pas nécessairement besoin de maîtriser le français ou l'anglais. [...] C'est un gros effort de s'ouvrir au marché du travail à l'extérieur. (Charlotte, 30, conseillère en réadaptation)

Dans de tels cas, l'enjeu de la maîtrise de la langue émerge. Toutefois, pour les conseillers en réadaptation, le plus important est de motiver les travailleurs à se rétablir, quitte à utiliser ce temps d'arrêt pour explorer un autre créneau d'emploi lorsque le lien est rompu.

\section{Stratégies pour contourner ou surmonter les obstacles à la réadaptation}

Il y a trois formes de retour au travail : a) la réintégration au poste occupé avant la lésion avec ou sans allègement de tâche, b) l'affectation temporaire pour réintégrer complètement le poste pré-lésionnel et c) un emploi convenable chez un autre employeur. Toutes ces options sont inscrites dans la LATMP, mais selon les conseillers en réadaptation et les travailleurs eux-mêmes, ces derniers n'ont pas un accès équitable aux mesures de réadaptation.

\section{Dresser une liste des postes réservés aux affectations temporaires}

Pour les conseillers en réadaptation, la présence d'un syndicat assure plus d'équité dans le traitement des demandes de réintégration au travail et accélère le processus :

C'est plus facile la prise en charge quand il y a un syndicat présent que quand il n'y a pas de syndicat. [...] C'est eux qui connaissent beaucoup plus l'entreprise que nous. [...] Comme pour enlever une tâche on va valider la proposition avec le syndicat et discuter avec lui [de] ce qui est possible. Il nous donne l'heure juste sur les conventions de travail, ça aide. [...] S'il n'y a pas de syndicat, c'est directement avec l'employeur, ça, c'est toujours plus difficile. [Dans de tels cas], on essaie de parler au superviseur, mais c'est moins facile. (Rosalie, 29, conseillère en réadaptation) 
Il y a moins de travailleurs immigrants que de travailleurs natifs du Québec qui occupent un poste dans une entreprise syndiquée ${ }^{6}$. Selon les répondants, au cours de leurs premières années d'insertion sur le marché du travail, ils cumulent des expériences en occupant des postes atypiques d'agence de placement, de surnuméraire ou de sous-traitant. Les entreprises clientes qui ont recours aux agences de location de personnel, même syndiquées, ne sont pas tenues de proposer un emploi convenable aux travailleurs occasionnels, puisqu'elles ne sont pas au sens de la loi l'employeur véritable, celui qui rémunère le travailleur.

Pour les entreprises non syndiquées, c'est fastidieux et non rentable de trouver un emploi convenable au travailleur en processus de réintégration ou de faire l'analyse d'un allègement des tâches. Certaines entreprises non syndiquées ont adopté une méthode expéditive pour convenir d'une affectation temporaire avec le travailleur et son conseiller en réadaptation :

[...] quand la personne est en CSST, dès qu'elle quitte, nous on lui donne une enveloppe d'assignations temporaires que l'on propose aux médecins: l'inspection visuelle, la pose d'étiquettes. Si le médecin juge qu'il y a arrêt de travail, on a des travaux légers pour qui veut se ramener au travail, le temps qu'il faudra. [...] les postes d'assignation temporaire on ne les donne pas aux travailleurs d'agences, parce que ce ne sont pas nos travailleurs. On va donner [aux travailleurs d'agence] les premiers soins. (représentant de l’Entreprise Gourmet-maison)

Selon l'un des cliniciens, il arrive que des employeurs contestent l'horaire des traitements lorsque ce dernier empiète sur l'horaire de travail. Ils réclament que les services de santé réservent les plages horaires de traitement de fins de journée aux travailleurs en affectation temporaire pour leur éviter de payer des heures perdues de travail.

Si le médecin ne prescrit pas de limitations par rapport au nombre d’heures [...] si le rendez-vous est à la fin de la journée, on lui faisait faire son 8 heures, puis on l'envoyait après ça au rendez-vous. Si c'était au milieu de la journée [...] comme à 14 heures, à 13 heures il a terminé sa journée, [...] on paye le 8 heures. (Entreprise Gourmet-maison)

Aux yeux des employeurs, l'affectation temporaire pour les travailleurs aux prises avec des douleurs chroniques est compliquée, soulève même dans certains cas de la suspicion, alors que le cumul d'exposition aux risques est bien réel :

[...] on a eu un cas, le travailleur a été à notre embauche passant un bout de temps via une agence de placement. [...] Quelques mois après sa permanence, il est tombé en accident de travail. (représentant de l'Entreprise Gourmet-maison)

Dans une usine, on n'a pas 15 millions de postes, la plupart des postes comportent des mouvements répétitifs. [...] C'est encore plus difficile quand le travailleur n'est pas collaborateur. (représentant de l'Entreprise CapGear)

Selon les conseillers en réadaptation, les employeurs semblent plus enclins à proposer un retour progressif qu'une affectation temporaire. La gestion du remplacement pour des journées complètes d'absence est plus simple que de répartir les tâches à compléter entre les travailleurs pleinement fonctionnels.

\section{Favoriser la mise à niveau des compétences des travailleurs immigrants}

Comme cela a été mentionné à plusieurs reprises par les personnes interrogées, les employeurs ne sont pas enclins à dresser une liste des emplois convenables ou à procéder à un allègement de tâches. C'est une obligation à laquelle les petites et les moyennes entreprises peuvent se soustraire, si le délai de réintégration dépasse le temps prévu par la loi au Québec (respectivement d'un an et de deux ans). Au-delà de ce délai, les services de réadaptation miseront sur la recherche d'un emploi convenable dans une autre entreprise. Or tout nouvel

\footnotetext{
${ }^{6}$ En 2012, au Québec, la couverture syndicale des natifs était de plus de $40 \%$, alors que chez les immigrants, elle était de $31,9 \%$, un taux de syndicalisation bien supérieur à celui constaté en Colombie-Britannique (15,5\%) et en Ontario (14,4\%), phénomène attribuable aux efforts des entreprises publiques à recruter des travailleurs immigrants (Boudarbat et Connolly, 2013).
} 
employeur peut légitimement exiger des nouvelles recrues qu'elles s'expriment couramment dans l'une ou l'autre des deux langues officielles du Canada (français et anglais).

Selon les conseillers en réadaptation, sans soutien, la réadaptation des travailleurs allophones est fastidieuse et peu concluante. Les conseillers sont souvent confrontés au dilemme suivant: déroger aux règles de l'emploi équivalent pour permettre aux travailleurs immigrants de retrouver un sens à leur rétablissement, pour qu'ils se réintègrent au marché du travail, ou les déclarer inemployables. Pour plusieurs conseillers interrogés, la solution passe par le compromis entre les besoins du marché du travail et la mise à niveau des compétences des travailleurs, en fonction du métier occupé antérieurement et de leurs compétences linguistiques. Pour élaborer une stratégie de compromis, il faut connaître le passé, le présent et les aspirations professionnelles du travailleur.

Avec l'immigrant, tu le sais petit bout par petit bout. Ça te prend beaucoup plus de temps à fouiller cette information-là, à arriver à créer un lien de confiance, à trouver les ressources. (Charlotte, 30, conseillère en réadaptation)

Il faut déterminer si le travailleur vit le processus de réadaptation comme un parcours déshonorant, voir litigieux, ou si l'arrêt de travail peut se transformer en une opportunité pour changer sa situation d'emploi.

\section{Discussion}

D'après nos résultats, les travailleurs immigrants rencontrent à la fois des difficultés courantes de réadaptation et des difficultés plus spécifiques liées à leur intégration sur le marché du travail québécois. Ces difficultés sont aggravées lorsque le processus de réadaptation se transforme en un "parcours déshonorant ", sans option pour améliorer les conditions de l'emploi prélésionnel. Éviter que le processus ne se transforme en parcours déshonorant ou litigieux pose un dilemme moral et administratif aux services publics. II faudrait assouplir l'interprétation de la règle de réintégration du travailleur dans un poste équivalent à celui occupé au moment de la lésion sans égard aux compétences et formations acquises antérieurement, assouplissement qui permettrait aux conseillers en réadaptation de proposer un juste compromis aux travailleurs pour qu'ils puissent briguer un poste qui reconnaitrait partiellement leurs compétences, même si celles-ci n'étaient pas requises pour l'emploi occupé au moment de la lésion. La reconnaissance complète des compétences n'est généralement pas possible sans procéder préalablement à une analyse des équivalences en se référant aux exigences canadiennes des métiers et des professions basées sur les cursus de cours et de stages attendus. Cette stratégie aurait pourtant le mérite d'apporter un sens à la quête de réintégration au travail.

Les travailleurs immigrants risquent d'aggraver leur situation, surtout en cas de troubles musculosquelettiques, en repoussant la déclaration de leur lésion aux limites du seuil de tolérance à la douleur avant de rapporter leur état de santé (Côté, Gravel, Dubé, Gratton et White., 2017), et ce, pour plusieurs raisons: par loyauté envers l'employeur, par crainte de perdre leur emploi ou de subir des représailles, ou encore de peur de s'enliser dans d'interminables litiges médico-légaux (Ahonen, Benavides et Benach, 2007; Benach, Muntaner, Chung et Benavides, 2010; European Agency for Safety and Health at Work, 2013; McCauley, 2005). La précarité du lien d'emploi va aussi les inciter à revenir plus tôt au travail pour éviter un congédiement.

Nos résultats sur la transformation du processus de réintégration au travail en " parcours de la honte » vont dans le même sens que ceux de Loisel et coll. (2005) et de Kristam et coll. (2016) en réadaptation et ceux en médiation interculturelle de la souffrance de Denizeau (2013) et Bouznah et Lewertowski (2014). La réadaptation des lésions professionnelles n'est possible que si elle prend en considération la souffrance vécue par le travailleur au sens physique, psychologique et symbolique et si elle s'inscrit dans un contexte social (Gratton, 2009). Or certains travailleurs immigrants se sont blessés dans un emploi de passage ou transitoire et ont espéré que cet arrêt de travail leur permette d'accéder à un emploi à la hauteur de leurs compétences. Loin de réaliser cet objectif, ils se sont retrouvés en quête d'un " emploi convenable » pour remplacer cet emploi de passage (Dubé et Gravel, 2014). II n'est donc pas étonnant que les travailleurs immigrants en situation de déqualification professionnelle au moment où ils ont subi une lésion vivent le processus de réadaptation à contresens de ce qu'ils attendaient de leur intégration. 
Cette étude exploratoire ne peut prétendre illustrer tous les cas de figure de la réadaptation des travailleurs immigrants ayant subi une lésion professionnelle. Pour obtenir une telle représentativité, nous aurions besoin d'une stratégie d'échantillonnage plus robuste : 1) inclure toutes les demandes de réadaptation adressées à la CNESST par des travailleurs issus de l'immigration au cours d'une année, 2) suivre tous les travailleurs pendant 24 mois, soit le temps maximal pour consolider une lésion - au-delà de cette période, on considère que l'amélioration de l'état de santé est peu significative, 3) prendre en considération toutes les options de programmes offerts (formation, francisation, mise à niveau des compétences acquises à l'étranger, etc.) pour comprendre les combinaisons efficaces de programmes menant à la réintégration durable sur le marché du travail. Notre étude, malgré ses limites méthodologiques, contribue à bonifier notre modèle d'analyse de la réadaptation, en tenant compte du contexte d’intégration des travailleurs immigrants sur le marché du travail.

Comme l'illustre la figure 2, le parcours migratoire et la situation de handicap au travail découlant d'une lésion professionnelle peuvent prendre différentes directions selon le niveau de scolarité du travailleur ou sa situation de surqualification. Ce parcours présente une discontinuité, une rupture du projet de vie initial, bien qu'il puisse, à l'inverse, comporter des opportunités de changement et de "reconstruction » (Charmaz, 2002). Cette représentation devrait pouvoir s'enrichir grâce à l'inclusion des variables médiatrices, dont les programmes adaptés à la situation de surqualification professionnelle des travailleurs.

\section{Figure 2. Enjeux de la discontinuité des parcours de réadaptation des travailleurs immigrants ayant subi une lésion professionnelle}

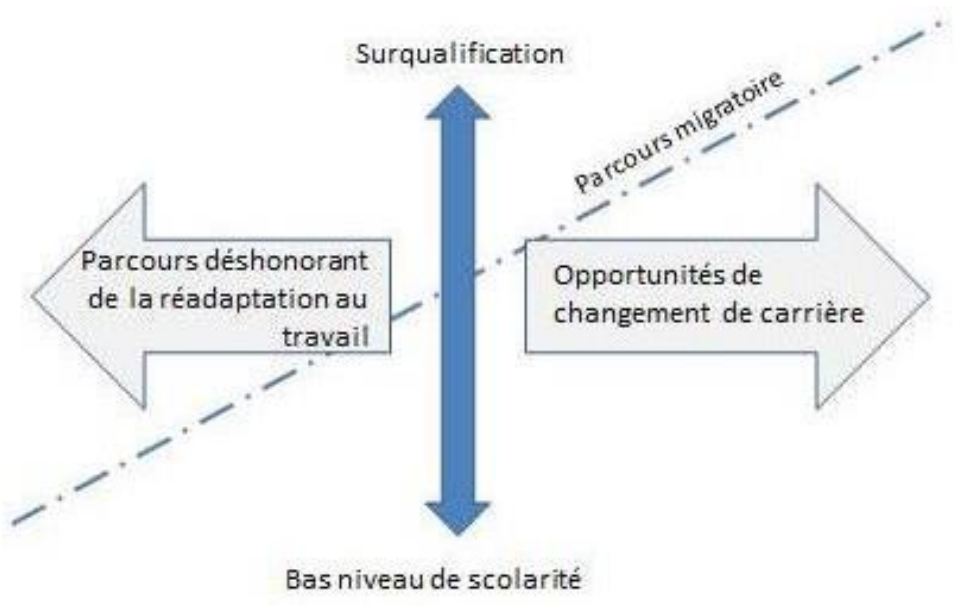

Pour les travailleurs immigrants, la réadaptation va au-delà de l'effort à surmonter la douleur envahissante, elle prend sa force dans les tentatives de transformer l'arrêt de travail en opportunité de changer une situation d'emploi, de réintégrer le travail avant la consolidation de l'état de santé. Si les modèles récents ont mis en lumière les effets du soutien de l'environnement social comme constituant des facteurs favorables à la réadaptation (Kristman et coll. 2016; Lederer et coll. 2014), le réseau de soutien demeure dissonant pour les travailleurs immigrants. Les amis, les collègues, le superviseur immédiat ou encore la direction des ressources humaines de l'entreprise, qui devraient être aux premières loges du soutien à la réadaptation (Lysaght et Larmour-Trode, 2008), sont cruellement absents auprès des travailleurs immigrants rencontrés, parce que ces derniers ont rarement des liens stables d'emploi, n'ont pas toujours leur famille à proximité et font rarement partie d'un collectif de travailleurs qui pourrait se montrer solidaire.

D'après nos résultats, trois types de problèmes émergent chez les travailleurs immigrants en processus de réadaptation : des problèmes de communication, de structures et d'équité. Chacun nécessite des aménagements de service distincts. Comme le suggèrent Dressler et Pils (2009), il est possible de suppléer au fardeau de la communication en implantant des programmes de contrôle de la douleur, avec des équipes de thérapeutes multiethniques et multilingues, pour développer des compétences à saisir le sens des mots, des symboles et des rôles invoqués lors des rencontres thérapeutiques en contexte interculturel (Sloots et coll., 2011). Selon Sadler, 
Wolfe et McKevitt (2014), ces programmes sont susceptibles d'améliorer la relation thérapeutique mais sont incapables de corriger le problème à la source, soit un traitement inéquitable des travailleurs ayant subi une lésion professionnelle en cas de de lien précaire d'emploi (Gravel et coll., 2016).

Certes, les services de réadaptation n'ont pas pour mission de corriger les inégalités de santé attribuables au travail et les conséquences de la flexibilité attendue de la main-d'œuvre. Toutefois, les services de réadaptation et surtout ceux de réintégration au travail pourraient élargir leur éventail d'offres de programmes en tenant compte de la dissonance d'un marché du travail non solidaire envers les travailleurs immigrants et en favorisant des structures d'accueil facilitant l'inclusion socioprofessionnelle. En ce sens, les programmes de mise à niveau des compétences linguistiques (Premji, 2015) et professionnelles devraient s'inscrire dans une approche populationnelle spécifique sur le plan sociétal, comme le proposent Lederer et coll. (2014).

\section{Conclusion}

Bien que tous les services publics subissent les contraintes de l'austérité administrative, il serait bénéfique de faire une analyse coûts-avantages de différents services de soutien à offrir aux travailleurs immigrants ayant subi une lésion professionnelle en fonction des finalités de leur réintégration sur le marché du travail. Quatre programmes pourraient être mis à l'étude : 1) les services de base (physiothérapie, ergothérapie et psychothérapie), 2) les services de base avec une mise à niveau des compétences linguistiques (apprentissage du français, de l'anglais), 3) les services de base avec une mise à niveau des compétences professionnelles acquises antérieurement et d) les services de base avec une mise à niveau des compétences linguistiques et professionnelles. L'analyse coûtsavantages pourrait comparer les dépenses d'investissements (soins, formations et ressources d'encadrement) et la finalité des résultats (rechute ou aggravation des lésions, réinsertion passagère ou durable sur le marché du travail). Un nouveau créneau de recherche centré sur les approches adaptées à la situation des travailleurs immigrants pourrait émerger et contribuer à l'avancement des connaissances en réadaptation.

\section{Références bibliographiques}

Ahonen, E., Benavides, F. et Benach, J. (2007). Immigrant populations, work and health-A systematic literature review. Scandinavian Journal of Work, Environment and Health, 33(2), 96-104.

Benach, J., Muntaner, C., Chung, H. et Benavides, F. (2010). Immigration, employment relations, and health: developing a research agenda. American Journal of Industrial Medicine, 53, 338-343.

Boudarbat, B. et Connolly, M. (2013). Évolution de l'accès à l'emploi et des conditions de travail des immigrants au Québec, en Ontario et en Colombie-Britannique entre 2006 et 2012. Série scientifique (2013s-28). Montréal : CIRANO (Centre interuniversitaire de recherche et d'analyse des organisations). https://www.cirano.qc.ca/pdf/publication/2013s-28.pdf

Bouznah, S. et Lewertowski, C. (2014). Quand les esprits viennent aux médecins : 7 récits pour soigner. Paris : In Press.

Charmaz, K. (2002). The Self as Habit: The Reconstruction of Self in Chronic Illness. Occupational Therapy Journal of Research : Occupation, Participation, and Health; 22(1): 31S-41S.

Commission des normes du travail au Québec (2012). Sondage visant à évaluer les conditions de travail des salariés temporaires d'agences de placement de personnel et les pratiques de celles-ci (Rapport d'analyse final). Dossier 79083-024. http://www.cnt.gouv.qc.ca/fileadmin/pdf/enquetes-et-recherches/Rapport-CNT79083024_nov_2012_VF.pdf

Côté, D., Gratton, D., Gravel, S. et Dubé, J. (2015). Les enjeux de la santé-sécurité du travail et les conditions de I'inclusion. Vie économique, $7(1), n^{\circ}$ spécial Immigration, diversité et inclusion : où en sommes-nous? http://www.eve.coop/?a=241

Côté, D., Gravel, S., Dubé, J., Gratton, D. et White, B. W. (2017). Comprendre le processus de réadaptation et de retour au travail dans le contexte des relations interculturelles (Rapport R-967). Montréal : IRSST. 
Côté, D. (2014). La réadaptation au travail des personnes issues de l'immigration et des minorités ethnoculturelles : défis, perspectives et pistes de recherche. Pistes, 16(2). http://pistes.revues.org/3633

Côté, D. et Coutu, M. F. (2010). A critical review of gender issues in understanding prolonged disability related to musculoskeletal pain: How are they relevant to rehabilitation? Disability and Rehabilitation, 32(2), 87-102.

Denizeau, L. (2013). L'expérience de la douleur, une activité symbolique ? Anthropologie et Santé (Revue internationale francophone anthropologie et santé), 7, 1-19. http://anthropologiesante.revues.org/1130

Dressler, D. et Pils, P. (2009). A qualitative study on cross-cultural communication in post-accident in-patient rehabilitation of migrant and ethnic patients in Austria. Disability and Rehabilitation, 31(14), 1181-1190.

Dubé, D. et Gravel, S. (2014). Les pratiques préventives auprès des travailleurs d’agences de location de personnel temporaire ou permanent : comparaison entre les travailleurs immigrants et non immigrants. Pistes, $\mathrm{n}^{\circ}$ spécial Travailleurs immigrants et santé et sécurité au travail, 16(2). http://pistes.revues.org/3631

Edelman, G. (2008 [1992]). Biologie de la conscience (trad. de l'anglais par A. Gershenfels). Paris : Odile Jacob.

European Agency for Safety and Health at Work (EU-OSHA). (2013). Priorities for occupational safety and health research in Europe: 2013-2020. Luxembourg : European Agency for Safety and Health at Work.

Gratton, D. (2009). L'interculturel pour tous. Une initiation à la communication pour le troisième millénaire. Anjou : Éditions Saint-Martin.

Gravel, S. et Dubé, J. (2016). Occupational health and safety for workers in precarious job situations: combating inequalities in the workplace. E-Journal of International Comparative Labour Studies, 5(3), 1-30. http://ejcls.adapt.it/index.php/ejcls_adapt/issue/view/47

Gravel, S., Legendre, G., Rhéaume, J., Séguin, G. et Gagné, C. (2013). Mesures de santé et de sécurité du travail dans les petites entreprises montréalaises embauchant une main-d'œuvre immigrante - Les stratégies favorables à la prise en charge. http://www.irsst.qc.ca/publications-et-outils/publication/i/100719/n/ mesures-de-sante-et-de-securite-du-travail-dans-les-petites-entreprises-montrealaises-embauchant-unemain-d-oeuvre-immigrante-les-strategies-favorables-a-la-prise-en-charge-r-793

Gravel, S., Vergara, D., Lippel, K., Dubé, J., Ducharme-Varin, J.-F. et Legendre, G. (2016). Santé et sécurité des travailleurs qui cumulent des précarités : la lutte aux inégalités de santé attribuables au travail. Montréal : Centre de recherche Léa-Roback sur les inégalités sociales de santé de Montréal. http://www.centrelearoback.org/assets/PDF/CLR-SylvieGravel_Rapport_Cumul_de_pecarite2016.pdf

Higgins, A., O'Halloran, P. et Porter, S. (2012). Management of long term sickness absence: A systematic realist review. Journal of occupational rehabilitation, 22, 322-332. https://link.springer.com/article/10.1007\%2Fs10926-012-9362-4

Kristman, V., Shaw, W.., Boot, C., Delclos, G., Sullivan, M. et Ehrhart, M. (2016). Researching complex and multilevel workplace factors affecting disability and prolonged sickness absence. Journal of occupational rehabilitation, 26, 399-416.

Lederer, V., Loisel, P., Rivard, M. et Champagne, F. (2014). Exploring the diversity of conceptualizations of work (dis)ability: A scoping review of published definitions. Journal of occupational rehabilitation, 24, 242-267.

Loisel, P., Buchbinder, R., Hazard, R., Keller, R., Scheel, I., van Tulder, M. et Webster, B. (2005). Prevention of work disability due to musculoskeletal disorders: The challenge of implementing evidence. Journal of occupational rehabilitation, 15(4), 507-524.

Lysaght, R. et Larmour-Trode, S. (2008). An exploration of social support as a factor in the return-to-work process. Work, 30, 255-266.

MacEachen, E., Kosny, A., Ferrier, S. et Chambers, L. (2010). the "toxic dose" of system problems: why some injured workers don't return to work as expected. Journal of occupational rehabilitation, 20, 349-366. 
Massé, R. (2016). Les travailleurs invisibles. Les risques pour la santé des travailleurs des agences de location de personnel (Rapport du directeur de la santé publique de Montréal 2016). Montréal : Direction régionale de santé publique, CIUSSS du Centre-Sud-de-l'île-de-Montréal.

http://www.dsp.santemontreal.qc.ca/fileadmin/documents/dossiers_thematiques/Autres_thematiques/Ra pport_2016/Rapdir_2016_FR_Web.pdf

McCauley, L. (2005). Immigrant workers in the United States: Recent trends, vulnerable populations, and challenges for occupational health. AAOHN Journal, 53(7), 313-319.

Niemeier, J., Burnett, D. et Whitaker, D. (2003). Cultural competence in the multidisciplinary rehabilitation setting: Are we falling short of meeting needs? Archives of physical medicine and rehabilitation, 84, 1240-1245.

Ockander, M. et Timpka, T. (2003). Women's experiences of long term sickness absence: implications for rehabilitation practice and theory. Scandinavian journal of public health, 31(2),143-148.

Ori, M. et Sargeant, M. (2011). Vulnerable workers; health safety and well-bewing. gower apllied research; introduction: ix-xiv.

Premji, S., Duguay, P., Messing, K. et Lippel, K. (2010). Are immigrants, ethnic and linguistic minorities overrepresented in jobs with a high level of compensated risk? Results from a Montréal, Canada study using census and workers' compensation data. American journal of industrial medicine, 53(9),875-885.

Premji, S. (2015). Barriers to return-to-work for linguistic minorities in Ontario: an analysis of narratives from appeal decisions. Journal of occupational rehabilitation, 25(2), 357-367.

Quinlam, M. (2011). We've been down this road before: vulnerable work and occupationnal health in historical perspective. Dans M. Sargeant et M. Giovannone (dir.), Vulnerable workers; health safety and well-bewing (p. 21-55). Farnham (R.-U.) : Gower Publishing.

Sadler, E., Wolfe, C. et McKevitt, C. (2014). Lay and health care professional understanding of self-management: a systematic review and narrative synthesis. Sage Open Medicine. sagepub.co.uk/journalsPermissions.nav. DOI: $10.1177 / 2050312114544493$

Schultz, I., Stowell, A., Feurstein, M. et Gatchel, R. (2007). Models of return to work for musculoskeletal disorders. Journal of occupational rehabilitation, 17, 327-352.

Sjöström, R., Melin-Johansson, C., Asplund, R. et Alricsson, M. (2011). Barriers to and possibilities of returning to work after a multidisciplinary rehabilitation programme. A qualitative interview study. Work: A journal of prevention, assessment and rehabilitation, 39, 243-250.

Sloots, M., Dekker, J., Bartels, E., Geertzen, J. et Dekker, J. (2011). Adaptations to pain rehabilitation for non-native patients with chronic pain. Disability and rehabilitation, 33(15-16), 1324-1329.

Sloots, M., Dekker, J., Pont, M., Bartels, E., Geertzen, J. et Dekker, J. (2010). Reasons of drop-out from rehabilitation in patients of Turkish and Moroccan origin with chronic low back pain in the Netherlands: A qualitative study. Journal of rehabilitation medicine, 42(6), 566-573.

Vosko, L. (2009). Less than adequate: regulating temporary agency work in the EU in the face of an internal market in services. Cambridge journal of regions, economy and society, 2(3), 395-411.

Vulture, M. et Provencher, Y. (2014). Les logiques de recours aux agences de travail temporaire et leurs pratiques de recrutement et de sélection de la main-d'œuvre. Dans J. Bernier et M. Vulture, Les agences de travail temporaire : leur rôle et leur fonctionnement comme intermédiaire du marché du travail ( $\mathrm{p}$ 39-66). Québec : Presses de l'Université Laval.

Waddell, G. (1987). Volvo Award in clinical sciences. A new clinical model for the treatment of low-back pain. Spine, $12,632-644$.

Young, A. (2010). Return to work following disabling occupational injury - facilitators of employment continuation. Scandinavian journal of work, environment and health, 36(6), 473-483. 